\title{
Protective Effects of Seabuckthorn Seed Oil against Acute Alcoholic-induced Liver Injury in Rats
}

\author{
Meihong $\mathrm{Xu}^{1}$, Na Wang ${ }^{2}$, Weirui Zhang ${ }^{2}$, Junbo Wang ${ }^{1, *}$ \\ ${ }^{1}$ Department of Nutrition and Food Hygiene, School of Public Health, Peking University, Beijing, PR China \\ ${ }^{2} \mathrm{R} \& \mathrm{D}$ center of TIENS Ltd., Tianjin, PR China \\ *Corresponding author: mrwang4j@163.com
}

Received October 25, 2014; Revised November 20, 2014; Accepted December 24, 2014

\begin{abstract}
The present study examined the protective effects of seabuckthorn seed oil (Hippophae rhamnoidesL seed oil., SBT) on acute alcoholic-induced hepatic damage in male SD rats. Our results showed that oral administration of SBT seed oil at doses of $0.167,0.333$ and $0.500 \mathrm{~g} / \mathrm{kg}$ for 45 days significantly reduced the changed levels of alanine aminotransferase (ALT) and aspartate aminotransferase (AST) in serum, and the level of triglyceride (TG) and malondialdehyde (MDA) in liver, which were induced by alcoholic-induced in rats. Moreover, the treatment of SBT seed oil was also found to significantly increase the activities of sglutathione peroxidase (GSHPx) in liver. Our study found that the optimal dose of SBT seed oil was $0.333 \mathrm{~g} / \mathrm{kg}$, as the minimum amount exhibiting the greatest hepatoprotective effects on alcoholic-induced liver injury, which have been supported by the evaluation of the liver histopathology in rats. In conclusion, it has been found the hepatoprotective effect of SBT seed oil at all tested doses.
\end{abstract}

Keywords: seabuckthorn seed oil, alcoholic-induced, liver, antioxidation

Cite This Article: Meihong Xu, Na Wang, Weirui Zhang, and Junbo Wang, "Protective Effects of Seabuckthorn Seed Oil against Acute Alcoholic-induced Liver Injury in Rats." Journal of Food and Nutrition Research, vol. 2, no. 12 (2014): 1037-1041. doi: 10.12691/jfnr-2-12-27.

\section{Introduction}

Seabuckthorn (SBT) is the popular medicinal plant and all parts of the plant are abundant in various bioactive substances, meanwhile it can be used as raw material for health foods and nutritional supplement [1]. SBT seeds oil, berries, leaves and bark are well known for their medicinal properties, suggested to be due to high contents of antioxidant substances. The leaf extracts of SBT, containing flavonoids, have been reported to have significant antioxidant, anti-bacterial, anti-viral, antiinflammatory, and anti-tumor activities [2]. SBT leaf extract has also hepatoparotective effects which might be due to its antioxidant activity [3]. Moreover, SBT berries have been reported to be a rich source of vitamins A, C, E, $\mathrm{K}$, flavonoids, carotenoids, organic acids, and oils $[4,5]$. Many medicinal effects of SBT berries have been reported, such as improving functions of digestive system and blood circulation [6]. Earlier studies have reported that SBT see oil contain high amount of unsaturated fatty acids, atocopherol, c-tocopherol, b-tocotrienol, carotenoids, and flavonoids, which are known to have significant antioxidant, anti-bacterial, anti-atherogenic, and cardioprotective activity $[7,8,9,10]$. SBT seed oil has protective effects against ischemic cerebral infarction and hypoxia induced transvascular fluid leakage in rats [11,12]. Furthermore, it have also been showed that the protective effects of SBT seed oil on carbon tetrachloride (CCl4)-induced hepatic damage in male ICR mice [13].

Alcohol and CCl4 are known as hepatotoxins sparked off liver injury and it is characterized by varying degrees of hepatocyte degeneration and cell death. It suggested that reactive oxygen species (ROS) including superoxide and hydroxyl radicals are known to play an important role in liver disease's pathology and progression as well as ROS have been proved to associate with the intoxication by alcohol. Moreover, alcohol has been commonly used for hepatotoxins in experimental hepatopathy.

In the present study, we hypothesized that administration of SBT seed oil may protect effects against alcoholicinduced hepatotoxicity in rats, through its enhancement of the antioxidant defense system. Therefore, male SD rats were orally treated with SBT seeds oil daily for 45 days. On Day 45, the rats were intragastrically administered alcohol. Hepatic TG, GSH-Px and MDA levels as well as activities of AST and ALT in serum were measured to monitor liver injury. The extent of alcoholic-induced liver injury was also analyzed through histopathological observations.

\section{Materials and Methods}

\subsection{Material}

Commercially available preparations of SBT form TIENS Ltd. (Tianjin, China) were wrapped in capsule 
prior to use. The quality of SBT seed oil is described and provided by the company. In accordance with the company provided data that the constituents in SBT seed oil include $98.29 \%$ crude fat, $0.93 \%$ crude protein, and $0.78 \%$ total carbohydrate. The major ingredients in SBT seed oil are linoleic acid and oleic acid.

Commercial kits used for the determination of GSH-Px and MDA were purchased form the Jiancheng Institute of Biotechnology (Nanjing, China). Ethanol and the other chemicals used were all of analytical grade and purchased from Beijing Chemical Co. (Beijing, China).

\subsection{Animals and Experimental Procedure}

A total of 72 male SD rats, weighing 180-220 g, were obtained from the Animal Service of Health Science Center, Peking University and adapted to the vivarium for one week before the treatment began. The conditions consisted of a filter-protected air-conditioned room, with a controlled temperature $\left(25 \pm 28^{\circ} \mathrm{C}\right)$, relative humidity (60 $\pm 5 \%$ ) and $12 \mathrm{~h}$ light/dark cycles (light on between 07:30 and 19:30 h). The animal treatment and maintenance were conducted in complete accordance with the Principle of Laboratory Animal Care (NIH Publication No. 85-23, revised 1985) and the guidelines of the Peking University Animal Research Committee.

All of the rats were fed with a model AIN-93M rodent diet (Vital River Limited Company, Beijing, China). The animals were randomly assigned to 6 groups, including a normal control group (defined as NC group), a solvent control group (defined as SC group), an alcohol control group, and 3 SBT intervention groups $(0.167,0.333$ and $0.500 \mathrm{~g} / \mathrm{kg} / \mathrm{BW}$ SBT seed oil, designated as the SLA, SMA and SHA groups).

SBT seed oil intervention started after the one week vivarium-adaption. The rats were intragastrically administered SBT seed oil whereas the NC group received an equal volume of distilled water, and the SC and AC group received an equal volume of edible peanut oil. The animal body weights were obtained twice/week to determine the effects of the SBT seed oil on the body weight and to adjust the injection volumes. The study lasted for 45 days. On Day 45, the rats were intragastrically administered 50\% alcohol (12 ml/kg*bw), except for the rats in NC group. At $16 \mathrm{~h}$ after the alcohol treatment, the rats were anesthetized by $\mathrm{CO} 2$ inhalation and then sacrificed. The blood was obtained, and the serum was separated by centrifugation at $3000 \mathrm{rpm}$ for 10 min; portions of the liver for histopathological examination were also obtained.

\subsection{Biochemical Assays}

The levels of alanine aminotransferase (ALT) and aspartate aminotransferase (AST) in the serum were detected using an automatic biochemistry analyzer (Hitachi 7020, Tokyo, Japan).

The triglycerides (TG), GSH-Px activity and the MDA contents in the liver were determined using detection kits, according to the manufacturer's respective protocols.

\subsection{Histological Study}

The livers were preserved in neutral buffered formalin and were processed for paraffin embedding, following the standard microtechnique. Four to five micron sections of livers stained with haematoxylin and eosin and then observed under microscope (Nikon, Y-FL light microscope, Japan).

\subsection{Statistical Analysis}

Statistical analyses were performed using SPSS software (version 18.0, SPSS Inc., Chicago, IL, USA). Variances in the measurement data were checked for homogeneity by Bartlett's test. When the data were homogeneous, the one-way analysis of variance test and multiple comparison of Dunnett's $t$ test were used. All reported $\mathrm{P}$ values were two-sided. A value of $\mathrm{P}<0.05$ was considered significant.

\section{Results}

\subsection{Effects of SBT on Body Weight}

Significantly higher body weight were observed in rats form the SBT seed oil group (at dose of 0.167, 0.333 and $\left.0.500 \mathrm{~g} / \mathrm{kg}^{*} \mathrm{bw}\right)$ as compared to the normal control $(\mathrm{P}<0.05)$ indicating that SBT seed oil could promote the increase of body weight. Alcohol administration caused a significantly increase of liver weight as well as liver to body weight, comparing with NC and SC group $(\mathrm{P}<0.05)$. Though remarkable differences had been shown on liver weight $(\mathrm{P}<0.05)$, there were no differences on liver to body weight, between the SBT-treated group and AC group ( $\mathrm{P}>0.05)$.

\subsection{Serum ALT and AST Levels}

The ALT and AST levels in serum were measured as an index of liver function. After alcohol administered, ALT level were increased in AC group. Inversely, AST level OF AC group were decreased. However, there were no differences has been shown among the groups $(\mathrm{P}>0.05)$.

\subsection{Liver TG, GSH-Px and MDA Levels}

We measured TG, MDA levels and the GSH-Px activities in the livers and the results are shown in Table 3. GSH-Px was measured as an index of antioxidant status of tissues. MDA level is widely used as a marker of free radical mediated lipid peroxidation injury.

The activities of liver GSH-Px in the AC group were decreased 25.8\%, compared with the NC group ( $\mathrm{P}>0.05)$. When compared with the AC group, the GSH-Px level were remarkable increased by $79.1 \%, 66.7 \%$ and $31.3 \%$, respectively, in the SBT seed oil treated group $(\mathrm{P}<0.05)$. On the contrary, MDA levels in AC group were significantly higher than that in NC group $(\mathrm{P}>0.05)$. The percentages of MDA levels in the SBT seed oil-treated group was significantly lower 28.8\%, 11.5 and $30.5 \%$ than that in AC group $(\mathrm{P}<0.05)$. In addition, compared with the AC group, the TG level were significantly lower in other groups $(\mathrm{P}<0.05)$. There were no significantly difference in statistics among the test doses of SBT seed oil. These findings indicated that the free radicals being released in the liver were effectively scavenged by SBT seed oil. Similar results were seen in the SC group. However, there were no significantly differences between the SC and AC group ( $\mathrm{P}>0.05)$. 


\subsection{Histopathological Observation}

Histopathological studies also provided an important evidence. The histological features of different groups were showed in Figure 1 (A: NC group; B: SC group; C: AC group; D: SAL group; E: SAM group; F: SAH group). There were no pathological changes in NC livers (Figure 1A). In SC and AC group, liver tissue pathological changed characterized by swelling and cells ranging irregularly. Also part of hepatocytes presented hepatic steatosis (Figure 1B, Figure 1C). Compared with the lesion observed in AC group, the lesions of SBT seed oil treated rats were of a much milder degree (Figure $1 \mathrm{D}$, Figure $1 \mathrm{E}$ and Figure 1F). These animals showed trace to mild diffused necrosis of hepatocytes, mild inflammatory cell infiltration, trace ballooning degeneration, and mild fibrosis.
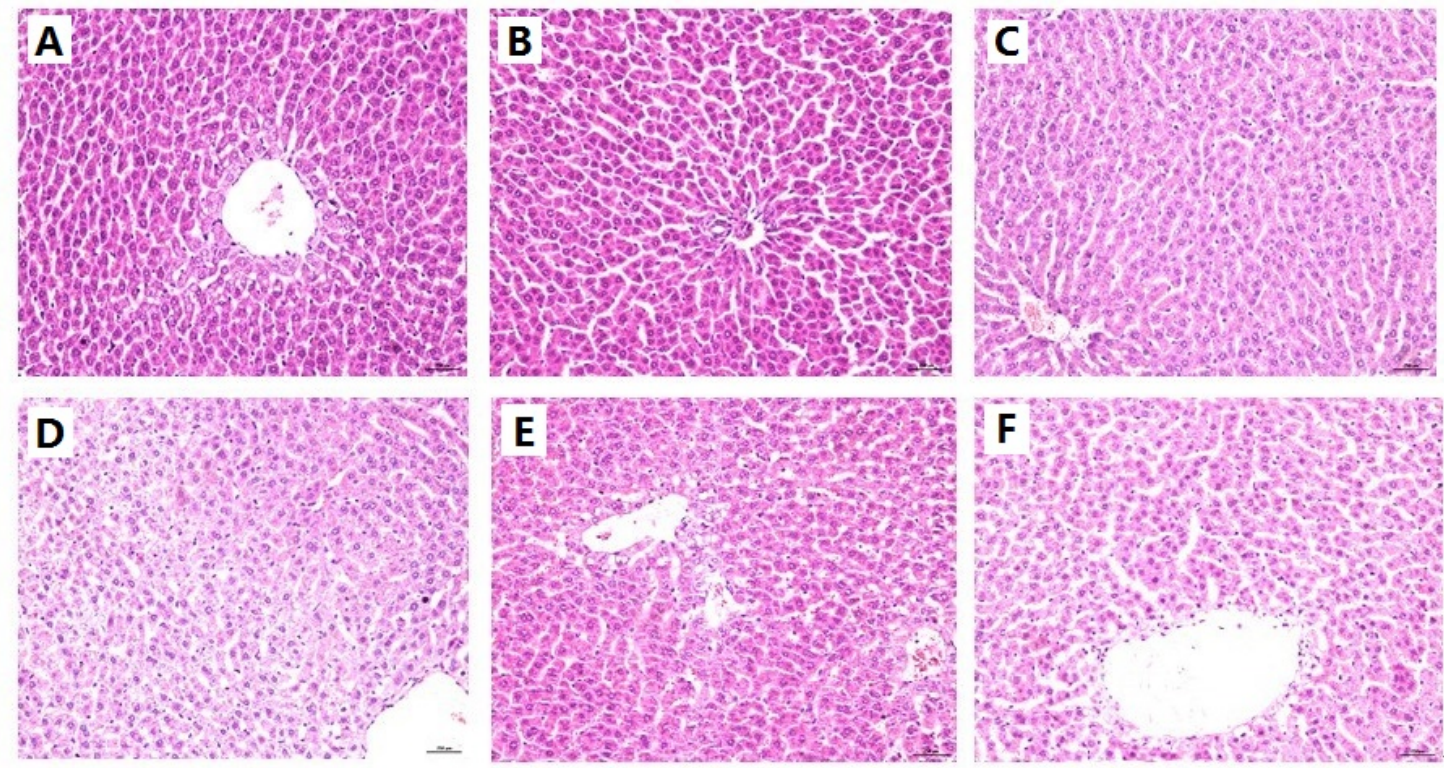

Figure 1. Representative histopathological changes of the liver in different groups (400 X, A: NC group; B: SC group; C: AC group; D: SLA group; E: SMA group; F: SHA group)

According to microscopic examinations, acute liver damages induced by alcohol was remarkably reduced by the administration of SBT seed oil, which was in good correlation with the results of the serum aminotransferase activities, hepatic antioxidant enzyme activities and hepatic lipid peroxidation.

Table 1. Effect of SBT seed oil on body and liver weight in alcoholic-induced rats

\begin{tabular}{|c|c|c|c|c|c|c|}
\hline Group & $\begin{array}{c}\text { Dose of SBT } \\
\left(\mathrm{g} / \mathrm{kg}^{*} \mathrm{bw}\right)\end{array}$ & $\mathrm{n}$ & $\begin{array}{l}\text { Baseline of body weight } \\
\text { (g) }\end{array}$ & $\begin{array}{l}\text { Body weight change } \\
\text { (g) }\end{array}$ & $\begin{array}{l}\text { Liver weight } \\
\text { (g) }\end{array}$ & $\begin{array}{c}\text { Liver/body } \\
(\%)\end{array}$ \\
\hline $\mathrm{NC}$ & 0 & 12 & $202.5 \pm 9.4$ & $85.3 \pm 18.4$ & $7.11 \pm 0.57 *$ & $2.48 \pm 0.25^{*}$ \\
\hline SC & 0 & 12 & $204.4 \pm 10.2$ & $107.2+26.7$ & $7.41 \pm 0.64^{*}$ & $2.39 \pm 0.24 *$ \\
\hline $\mathrm{AC}$ & 0 & 12 & $200.3 \pm 8.5$ & $95.2 \pm 19.7$ & $8.42+0.83$ & $2.85 \pm 0.22$ \\
\hline SAL & 0.167 & 12 & $203.8 \pm 9.7$ & $261.0 \pm 39.3^{*}$ & $13.15 \pm 1.67 *$ & $2.83 \pm 0.24$ \\
\hline SAM & 0.333 & 12 & $204.2+7.7$ & $253.7 \pm 30.7^{*}$ & $13.00 \pm 0.96 *$ & $2.84 \pm 0.19$ \\
\hline $\mathrm{SAH}$ & 0.500 & 12 & $204.1 \pm 8.6$ & $260.4 \pm 17.5^{*}$ & $12.88 \pm 0.91 *$ & $2.77 \pm 0.18$ \\
\hline
\end{tabular}

Values are present by mean \pm SD.

$* P<0.05$ compare with AC group.

Table 2. Effect of SBT seed oil on serum ALT and AST level in alcoholic-induced rats

\begin{tabular}{ccccc}
\hline Group & $\begin{array}{c}\text { Dose of SBT } \\
(\mathrm{g} / \mathrm{kg} \text { (bw) }\end{array}$ & $\mathrm{n}$ & $\begin{array}{c}\text { ALT } \\
(\mathrm{U} / \mathrm{L})\end{array}$ & $\begin{array}{c}\text { AST } \\
(\mathrm{U} / \mathrm{L})\end{array}$ \\
\hline NC & 0 & 12 & $42.9 \pm 11.9$ & $185.1 \pm 36.8$ \\
SC & 0 & 12 & $46.0 \pm 8.4$ & $180.6 \pm 45.4$ \\
AC & 0 & 12 & $43.2 \pm 9.8$ & $155.2 \pm 25.8$ \\
SAL & 0.167 & 12 & $52.5 \pm 10.4$ & $145.1 \pm 23.7$ \\
SAM & 0.333 & 12 & $55.0 \pm 13.7$ & $144.2 \pm 23.0$ \\
SAH & 0.500 & 12 & $50.5 \pm 9.1$ & $142.6 \pm 15.5$ \\
\hline
\end{tabular}

Values are present by mean \pm SD.

$* P<0.05$ compare with AC group.

Table 3. Effect of SBT seed oil on liver TG, GSH and MDA level in alcoholic-induced rats

\begin{tabular}{ccccc}
\hline Group & $\begin{array}{c}\text { Dose of SBT } \\
\text { (g/kg*bw) }\end{array}$ & $\mathrm{n}$ & $\begin{array}{c}\text { TG } \\
\text { (mmol/g) }\end{array}$ & $\begin{array}{c}\text { GSH-Px } \\
\text { (mg/gprot) }\end{array}$ \\
\hline NC & 0 & 12 & $0.41 \pm 0.17^{*}$ & $0.53 \pm 0.24$ \\
SC & 0 & 12 & $0.52 \pm 0.10^{*}$ & $0.64 \pm 0.16$ \\
AC & 0 & 12 & $0.75 \pm 0.19$ & $0.48 \pm 0.15$ \\
SAL & 0.167 & 12 & $0.44 \pm 0.15^{*}$ & $0.86 \pm 0.48^{*}$ \\
SAM & 0.333 & 12 & $0.52 \pm 0.19^{*}$ & $0.80 \pm 0.30^{*}$ \\
SAH & 0.500 & 12 & $0.46 \pm 0.20^{*}$ & $0.63 \pm 0.16$ \\
\hline
\end{tabular}

Values are present by mean \pm SD.

$* P<0.05$ compare with AC group. 
Table 4. Effect of SBT seed oil on hepatic histopathology of liver damage in rats treated with alcohol

\begin{tabular}{cccc}
\hline Group & $\begin{array}{c}\text { Dose of SBT } \\
(\mathrm{g} / \mathrm{kg} \text { *bw) }\end{array}$ & $\mathrm{n}$ & hepatic steatosis \\
\hline NC & 0 & 10 & $1.3 \pm 0.7$ \\
SC & 0 & 10 & $1.0 \pm 0.5^{*}$ \\
AC & 0 & 10 & $1.7 \pm 0.7$ \\
SAL & 0.167 & 10 & $1.4 \pm 0.5$ \\
SAM & 0.333 & 10 & $0.4 \pm 0.5^{*}$ \\
SAH & 0.500 & 10 & $0.1 \pm 0.3^{*}$ \\
\hline
\end{tabular}

Values are present by mean $\pm \mathrm{SD}$.

$* P<0.05$ compare with AC group.

\section{Discussion}

SBT seed oil contain high amounts of tocotrienols, carotenoids, and flavonoids, which are known to have significant antioxidant activity. It has shown that SBT seed oil had changed the activities of AST and ALT in serum and markedly checked the depletion of GSH-Px in liver of rats induced by hepatotoxins of acetaminophen and CCl4 $[13,14]$. Therefore, we considered that SBT seed oil is useful in the prevention of various hepatic damages induced by oxidative stress. In the present study, the capability of SBT seed oil to protect against acute alcoholic-induced hepatotoxicity and oxidative stress was investigated.

Many studies have shown that a crucial mechanism of the hepatoprotective effects may be related to the antioxidant capacities to scavenge reactive oxygen species. In fact, a considerable body of literature has reported that numerous antioxidant agents, such as vitamin $\mathrm{E}$, Bupleurum kaoi Liu extract, saponins from Platycodon grandiflorum roots extracts and Dunaliella salina reduce alcoholic-induced hepatotoxicity effects by prevention of lipid peroxidation. In the present study, we found that treatment with the SBT seed oil significantly inhibits alcoholic-induced liver damage as evidenced by decreased serum activities of AST and ALT. The results of reduction TG effects on alcoholic-induced liver damages in rats of treatment with SBT seed oil could be attributed to the relatively high content of unsaturated fatty acids in SBT seed oil. In fact, about $80 \%$ of the total fatty acids present in the SBT seed oil are unsaturated fatty acids and the predominant unsaturated fatty acids are oleic acid, linoleic acid, and linolenic acid.

To prevent the oxidative damage, tissues have constructed an antioxidant defense system that includes non-enzymatic antioxidants and enzymatic antioxidants. Therefore, the enzymatic antioxidants activities and/or the inhibition of free radicals generation are important in terms of protecting the liver from alcoholic-induced damage. GSH-Px metabolizes $\mathrm{H}_{2} \mathrm{O}_{2}$ and hydroperoxides to non-toxic products and terminates the chain reaction of lipid peroxidation by removing lipid hydroperoxides from the cell membrane. These antioxidant enzymes are effortlessly inactivated by lipid peroxides or free radical, which results in decreased activities of these enzymes in alcoholic Toxicity. The results of the present study indicate that GSH-Px was significantly elevated by administration of SBT seed oil to alcoholic-intoxicated rats, suggesting that it has the ability to restore/maintain the activity of hepatic enzymes in alcoholic-damaged liver. The similar results in other research confirmed that SBT seed oil played a protective role and blocked depletion of the hepatic GSH-Px in acetaminophen-induced and CCl4induced liver damage.

Lipid peroxidation by free radical derivatives of alcohol is one of the principal mechanisms of alcoholic-induced liver damage. In experimentation, MDA is a major reactive aldehyde that reveals during the peroxidation of biological membrane polyunsaturated fatty acid. The elevation of MDA levels in the liver imply enhanced peroxidation leading to tissue damage and breakdown of the antioxidant defense mechanisms to prevent the formation of superabundant free radicals. In the present study, alcoholic-induced toxicity caused an increase in MDA levels of liver tissue. By contrast, treatment with SBT seed oil significantly reversed these changes. SBT seed oil administration caused a significant decrease in MDA levels as compared to the alcoholic-induced toxicity group, suggesting that SBT seed oil could protective against from the alcoholic-induced lipid peroxidation in rats.

In histological examination of this study, liver damages were evaluated including hepatocyte necrosis, inflammatory cell infiltration, and ballooning degeneration by haematoxylin and eosin stain. Histological examination of rats' liver treated with alcoholic shows significant hepatotoxicity characterization, such as necrosis in hepatic lobules, inflammatory infiltration of lymphocytes, hypertrophy, Kupffer cells around the central vein, and fibrosis. However, administration with SBT seed oil significantly decreased these hepatotoxicity characterization in animals' liver, suggesting that SBT seed oil has a protection against alcoholic-induced liver injury.

Through the estimations of this study, the three test doses of SBT seed oil exhibited good hepatoprotective effects on alcoholic-induced liver damages in rats, but there did not increase of the hepatoprotective effects with the dose increase. Therefore, the results suggest that the dose of $0.333 \mathrm{~g} / \mathrm{kg}^{*} \mathrm{bw}$ of SBT seed oil exhibits optimal hepatoprotective effects on alcoholic-induced liver damage

\section{Conclusions}

In conclusion, this is the first study demonstrate that treatment of SBT seed oil were effective in prevention of alcohol-induced hepatic damage in SD rats. Among test doses of SBT exhibits hepatoprotective effects on alcoholinduced liver damages and we suggest that treatment with $0.333 \mathrm{~g} / \mathrm{kg}$ *bw of SBT exhibited optimal protection on the alcohol-induced hepatotoxicity and the good hepatoprotective effects.

\section{Acknowledgement}

The authors gratefully thank TIENS Ltd. for providing the samples used in this study.

\section{References}

[1] Shi, H.L., Cai, H.J., Chen, X.Y., Yang, C.M. Studies on the antioxidative effects of Hippophae rhamnoidesL. seed oil. Acta Nutr. Sin, Vol. 1994; 3: 292-5 (in Chinese). 
[2] Ganju, L., Padwad, Y., Singh, R., Karan, D., Chanda, S., Chopra, M.K., Bhatnagar, P., Kashyap, R., Sawhney, R.C. Antiinflammatory activity of seabuckthorn (Hippophae rhamnoides) leaves. Int. Immunopharmacol. 2005; 5(12): 1675-84.

[3] Geetha, S., Jayamurthy, P., Pal, K., Pandey, S., Kumar, R., Sawhney, R.C. Hepatoprotective effects of seabuckthorn (Hippophae rhamnoidesL.) against carbon tetrachloride induced liver injury in rats. J. Sci. Food Agric. 2008; 88(9): 1592-97.

[4] Pintea, A., Marpeau, A., Faye, M., Socaciu, C., Gleizes, M. Polar lipid and fatty acid distribution in carotenolipoprotein complexes extracted from seabuckthorn fruits. Phytochem. Anal. 2001; 12(5): 293-8.

[5] Kallio, H., Yang, B., Peippo, P. Effects of different origins and harvesting time on vitamin $\mathrm{C}$, tocopherols and tocotrienols in seabuckthorn (Hippophae rhamnoides) berries. J. Agric. Food Chem, 2002a; 50(21): 6136-42.

[6] Yang, B., Kallio, H. Composition and physiological effects of seabuckthorn (Hippophae) lipids. Trends Food. Sci. Tech. 2002; 13: 160-7.

[7] Yang, B., Karlsson, R., Oksman, P., Kallio, H. Phytosterols in seabuckthorn (Hippophae rhamnoidesL.) berries: identification and effects of different origins and harvesting times. J. Agric. Food Chem. 2001; 49(11): 5620-9.

[8] Kallio, H., Yang, B., Peippo, P., Tahvonen, R., Pan, R. Triacylglycerols, glycerophospholipids, tocopherols, and tocotrienols in berries and seeds of two subspecies (ssp. sinensis and ssp. mongolica) of seabuckthorn (Hippophae rhamnoides). J. Agric. Food Chem. 2002b; 50(10): 3004-9.

[9] Negi, P.S., Chauhan, A.S., Sadia, G.A., Rohinishree, Y.S., Ramteke, R.S. Antioxidant and antibacterial activities of various seabuckthorn (Hippophae rhamnoidesL.) seed extracts. Food Chem. 2005; 92: 119-24.

[10] Basu, M., Prasad, R., Jayamurthy, P., Pal, K., Arumughan, C., Sawhney, R.C. Anti-atherogenic effects of seabuckthorn (Hippophaea rhamnoides) seed oil. Phytomedicine. 2007; 14(11): 770-7.

[11] Berton, T.R., Conti, C.J., Mitchell, D., Cheng, T.J., Wang, Y.B., Gao, L.P., Sun, Y.F., Zhang, J. The protection of seed oil ofHippophae rharmnoideson ischemic cerebral infarction in rats", Zhongguo Zhong Yao Za Zhi, 2003; 28: 548-50 (in Chinese).

[12] Purushothaman, J., Suryakumar, G., Shukla, D., Malhotra, A.S., Kasiganesan, H., Kumar, R., Sawhney, R.C., Chami, A. Modulatory effects of seabuckthorn (Hippophae rhamnoidesL.) in hypobaric hypoxia induced cerebral vascular injury. Brain Res. Bull. 2008; 77(5): 246-52.

[13] Hsu, Y.W., Tsai, C.F., Chen, W.K., Lu, F. J. Protective effects of seabuckthorn (Hippophae rhamnoides L.) seed oil against carbon tetrachloride-induced hepatotoxicity in mice. Food and Chemical Toxicology 2009; 47(9): 2281-8.

[14] Cheng, T.J., Pu, J.K., Wu, L.W., Ma, Z.R., Cao, Z., Li, T.J. An preliminary study on hepato-protective action of seed oil ofHippophae rhamnoidesL. (HR) and mechanism of the action. Zhongguo Zhong Yao Za Zhi. 1994; 19: 367-70 (in Chinese). 